\title{
Multiple Equilibria for Cross-Gyre Flow between Subpolar and Subtropical Gyres
}

\author{
RICHARD SCHOPP \\ Laboratoire de Physique des Océans, CNRS IFREMER UBO, Universite de Bretagne Occidentale, Brest, France
}

(Manuscript received 27 May 1992, in final form 22 October 1992)

\begin{abstract}
A simple Sverdrup-type two-layer model that allows the outcropping of isopycnals is forced by wind stress, is completed with a frictional western boundary layer, and is investigated along the zero wind-stress curl line separating the subpolar gyre from the subtropical gyre. The study focuses on the different cross-gyre flow patterns. Intermediate length-scale dynamics, which is able to take the dispersion of Rossby waves and the steepening of isopycnals into account, is used to analyze the evolution of these cross-gyre currents. In particular, these transients show that the solution, which exhibits an arrested Rossby wave, is unstable in the western part of the basin. Nevertheless, this solution is able to evolve to other more stable solutions present in the dynamics: one in which there is an exchange of water masses between gyres and another one in which both gyres are independent. The first one has a deep (upper) slow northward (southward) flow in midoceanic regions and a strong western deep (upper) southward (northward) boundary current. This current system could well help to account for some of the transport in the western boundary undercurrent observed in the North Atlantic Ocean, and therefore the theory presented could indicate that the undercurrent and cross-gyre flow might have wind-driven components. The second stable solution, in which exchange is not allowed, would be rather representative of the North Pacific Ocean.
\end{abstract}

\section{Introduction}

The vertical structure of the wind-driven large-scale circulation has been examined in a number of recent models (Rhines and Young 1982; Luyten et al. 1983, hereafter LPS). A common feature of these inviscid wind-driven oceanic models is that the different gyres are nearly unable to exchange water masses across their common boundaries. Since Sverdrup dynamics requires a vanishing net meridional transport along the zero wind-stress curl line (denoted by $\Gamma$ ), barotropic cross-gyre flow is not allowed. Furthermore, since vertical velocities are continuous with depth in these models, baroclinic circulation with opposite currents in two adjacent layers must satisfy highly restrictive conditions. Indeed, Pedlosky (1984) and Schopp and Arhan (1986) have extracted singular solutions from the ventilated thermocline equations that are able to exchange water between the gyres. Their solutions, valid only away from boundary layers, presented a noteworthy character being that of an arrested baroclinic Rossby wave by a barotropic zonal-mean current along the boundary between the subpolar and subtropical gyres where Ekman pumping vanishes (Schopp 1988). A lack in these Sverdrup-type solutions was their inability to resolve the western boundary layer matching smoothly to the interior solution. The present work

Corresponding author address: Dr. Richard Schopp, Laboratoire de Physique des Océans, UBO/Faculté des Sciences et Techniques, 6, avenue Le Gorgeu, B.P. 452, 29275 Brest Cedex, France. will therefore complement the previous studies on cross-gyre flow in including such boundary layers. In adding friction to close the circulation, one is able to extend the arrested wave solution up to the western coast. This steady current pattern exhibits a slow northward flow in the deeper layer completed by a southward return flow on the western coast, a flow pattern similar to the observed North Atlantic one. The wind being the forcing of this flow pattern, the theory presented brings up a mechanism able to explain the deep water-mass circulation: a mechanism that could complement the thermohaline circulation developed by Stommel and Arons (1960). Nevertheless, this particular solution presents an unstable character and it is shown that it is situated at the boundary of two more stable solutions. One of them is close to the aforementioned arrested solution with a similar flow pattern, and the other one is without water mass exchange between gyres. Transients are computed to analyze the behavior of these flow patterns and to deduce the states toward which this solution evolves.

The dynamics used to carry out this study allow large vertical excursions of density surfaces and the dispersion of Rossby waves. The study will focus only on the gyre separation line.

Section 2 presents the dynamical equations, section 3 some physical behavior inside the equations, while in section 4 the steady and transient solutions are analyzed. In section 5 the western barotropic boundary layer is shifted eastward into the basin's interior giving rise to baroclinic interior layers and warm and cold 
ring-type features. Finally, cross-isopycnal upwelling is included to access the cross-gyre flow pattern.

\section{Dynamics}

A two-layer model is considered (Fig. 1) with $\Delta \rho$ as the density jump. The flow in both layers is supposed to be forced by wind only. The study will focus on the zero wind-stress curl line $\Gamma$ and assume the interior of the ocean to be inviscid. Outcropping of the deep layer is assumed at higher latitudes where Ekman pumping plays the role of a potential vorticity "source" and forces planetary motion in this layer farther south (like in Schopp and Arhan 1986). Dissipative processes will be active only in western boundary layers. The dynamics used is of intermediate scale type-dynamics able to take a large span of length scale into account, ranging from the Rossby deformation radius to the planetary scale (Charney and Flierl 1981). The time scale is chosen to give order 1 propagation of long baroclinic Rossby waves; that is, $\beta L_{D}^{2} /(\omega L)=\mathrm{O}(1)$, where $\beta$ is the meridional variation of the Coriolis parameter, $L_{D}$ $=\left(g^{\prime} H_{1} H_{2} / H\right)^{1 / 2} / f$ the Rossby deformation radius, $H_{1}$ and $H_{2}$ are the upper- and lower-layer mean depths, $H=H_{1}+H_{2}, \omega$ is an inverse of a time scale $(1 / \omega$ is of the order of one year), $g^{\prime}=g \Delta \rho / \rho$ is the reduced gravity; whereas the length scale will be taken of intermediate scale, that is, greater than the deformation radius but smaller than the earth radius $(L=100$ $1000 \mathrm{~km}$ ). This scaling allows us to keep both a large vertical excursion of isopycnals and Rossby wave dispersion.

The nondimensionalized inviscid vorticity equations for barotropic and baroclinic modes in this two-layer model with the aforementioned dynamics are given by

$$
\begin{gathered}
\psi_{b x}+F_{D}^{-1} \nabla^{2} \psi_{b t}-\frac{\epsilon}{\beta_{0}} J\left(\nabla^{2} \psi_{b}, \psi_{b}\right)-\frac{\epsilon H_{1} H_{2}}{\beta_{0} H^{2}} J\left(\nabla^{2} \psi_{c}, \psi_{c}\right)=\frac{w_{e}}{\epsilon \beta_{0} f H} \\
\psi_{c t}-\left[1+\epsilon F_{D} \frac{H_{1}-H_{2}}{H} \psi_{c}-\left(\epsilon F_{D}\right)^{2} \frac{H_{1} H_{2}}{H^{2}} \psi_{c}^{2}\right] \psi_{c x}-F_{D}^{-1} \nabla^{2} \psi_{c t}+\frac{\epsilon}{\beta_{0}}\left[J\left(\nabla^{2} \psi_{b}, \psi_{c}\right)+J\left(\nabla^{2} \psi_{c}, \psi_{b}\right)\right.
\end{gathered}
$$

$$
\left.+\frac{H_{1}-H_{2}}{H} J\left(\nabla^{2} \psi_{c}, \psi_{c}\right)\right]-\frac{\epsilon F_{D}}{\beta_{0}} J\left(\psi_{c}, \psi_{b}\right)-\frac{F_{D} w_{e}}{\beta_{0} f H} \psi_{c}=\frac{w_{e}}{\epsilon \beta_{0} f H_{1}},
$$

[III]

where $\psi_{b}=\psi+H_{2} / H \psi_{2}+F_{D} H_{1} H_{2} /\left(2 H^{2}\right) \psi_{2}^{2}$ represents the barotropic mode while $\psi_{c}=\psi_{2}$ the baroclinic one, $\psi$ and $\psi_{2}$ are the sea surface and the interface displacements. The barotropic mode has been defined by canceling the baroclinic one $\left(v_{2}-v_{1}=0\right)$ and the baroclinic one by canceling the barotropic mode $\left(\mathrm{H}_{2} v_{2}\right.$ $\left.=-H_{1} v_{1}\right)$.

For more details about the derivation and the scaling of these equations refer to Schopp (1988); Eqs. (2.1a,b) are obtained from Eqs. (3.9) and (3.10) in his paper by replacing $\eta$ with $\psi, \eta_{2}$ with $\psi_{2}$ and rewriting equations in function of $\psi_{b}$ and $\psi_{c}$. The subscripts $x, y, z$, and $t$ denote partial differentiation.

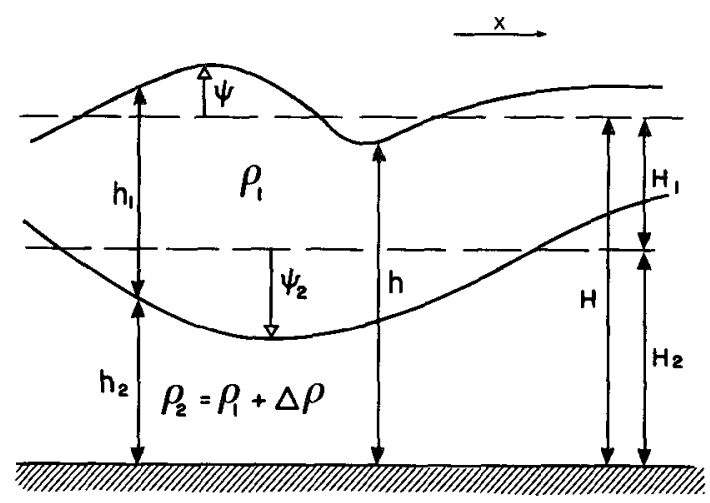

FIG. 1. A schematic cross section of the two-layer model.
Thicknesses have been parameterized as follows: $h$ $=H(1+\epsilon F \psi) ; h_{2}=H\left[H_{2} / H+\epsilon F_{D}\left(H_{1} H_{2} / H^{2}\right) \psi_{2}\right]$; $h_{1}=h-h_{2}\left[F=L^{2} / L_{E}^{2}\right.$ being the external Froude number with $\left.L_{E}=(g H)^{1 / 2} / f\right) ; \epsilon=U /(f L)$ being the Rossby number; $F_{D}=L^{2} / L_{D}^{2}$ the internal Froude number; $\beta_{0}=\beta L / f$ is the planetary parameter; and $w_{e}$ the Ekman pumping].

For simplicity the advection of relative vorticity will be neglected; that is, all terms in $\epsilon / \beta_{0}$ are considered small compared to any physical parameter kept.

The nonlinear Rossby wave phase speed [ first term in brackets in (2.1b)] behavior comes from the fact that $h_{1}$ and $h_{2}$ are able to vary in the phase speed itself since large excursions of isopycnals are allowed; the phase speed is given by the first term in brackets in (2.1b), which is the nondimensionalized form of $c_{R}$ $=\beta g^{\prime} h_{1} h_{2} /\left(f^{2} h\right)$. Physically the stretching term is great enough to add a space- and time-dependent background vorticity field on which Rossby waves propagate. The Rossby wave phase speed, being proportional to the product of $h_{1}$ and $h_{2}$, can be decomposed in a function of $\psi_{2}$ showing the appearance of the linear and quadratic terms in the preceding expression [terms $I$ and II in (2.1b)] (Dewar 1987).

The physical difference between Eqs. (2.1a,b) and quasigeostrophic dynamics is the added nonlinear part of the propagation speed [I and II in $(2.1 \mathrm{~b})]$ and a term where the forcing is corrected by the upper-layer 
thickness [III in $(2.1 \mathrm{~b})$ ], both terms being consequences of allowing large excursion of isopycnals. If the advection of relative vorticity is neglected, $(2.1 \mathrm{a}, \mathrm{b})$ reduce to

$$
\psi_{b x}+F_{D}^{-1} \nabla^{2} \psi_{b t}=\frac{w_{e}}{\epsilon \beta_{0} f H}-R_{s} \nabla^{2} \psi_{b}+R \nabla^{4} \psi_{b}
$$

(i)

$$
\begin{gathered}
\psi_{c t}-\left[1+\epsilon F_{D} \frac{H_{1}-H_{2}}{H} \psi_{c}\right. \\
\left.-\left(\epsilon F_{D}\right)^{2} \frac{H_{1} H_{2}}{H^{2}} \psi_{c}^{2}+\frac{\epsilon F_{D}}{\beta_{0}} \psi_{b y}\right] \psi_{c x} \\
=F_{D}^{-1} \nabla^{2} \psi_{c t}-\frac{\epsilon F_{D}}{\beta_{0}} \psi_{b x} \psi_{c y}+\frac{w_{e}}{\epsilon \beta_{0} f H_{1}} \\
\quad+\frac{F_{D} w_{e}}{\beta_{0} f H} \psi_{c}+r_{s} \nabla^{2} \psi_{c}
\end{gathered}
$$

where $\psi_{b x}$ and $-\psi_{b y}$ are the meridional and zonal barotropic flow.

Friction is included to close the circulation on the western boundary of the basin. In the barotropic field either a Rayleigh friction-type western boundary layer $\left(R_{s}\right)$ or a Munk-type one $(R)$ is added to the dynamics, whereas in the baroclinic field a Rayleigh boundary layer $\left(r_{s}\right)$ is assumed for simplicity.

In the baroclinic equation, the barotropic velocity can be evaluated from the barotropic mode. Integrated, Eq. (2.2a) gives formally

$$
\begin{aligned}
\psi_{b}=-\int_{x}^{x_{e}} w_{e} /\left(\epsilon \beta_{0} f H\right) d x^{\prime} \\
\quad-F_{D}^{-1} \int_{x} \nabla^{2} \psi_{b t} d x^{\prime}+\int_{x} \text { friction } d x
\end{aligned}
$$

Substituted into the baroclinic equation, the term exhibiting dispersion (i) will appear of order $\epsilon / \beta_{0}$, which is of the same order as the advection of relative vorticity and therefore will be neglected. As the barotropic equation is decoupled from the baroclinic one and since we shall deal with a steady wind field, we shall suppose the barotropic mode steady and neglect the dispersive term (i) in (2.2a).

Since the common boundary between the subtropical and subpolar gyres is of interest, attention will be focused on $\Gamma$ (where $w_{e}=0$ but $\partial w_{e} / \partial y>0$ ). For process studies dispersive $y$ terms will be neglected in these equations. Equations $(2.2 \mathrm{a}, \mathrm{b})$ along this line reduce then to

$$
\begin{aligned}
& \psi_{b x}=\frac{w_{e}}{\epsilon \beta_{0} f H}-R_{s} \psi_{b x x}+R \psi_{b x x x x}, \quad w_{e}=0 \\
& \psi_{c l}-\left[-c_{R}-U_{b}(x)\right] \psi_{c x}-F_{D}^{-1} \psi_{c x x t}=r_{s} \psi_{c x x},
\end{aligned}
$$

where the Rossby wave speed is given by

$$
c_{R}=-1-\epsilon F_{D} \frac{H_{1}-H_{2}}{H} \psi_{c}+\left(\epsilon F_{D}\right)^{2} \frac{H_{1} H_{2}}{H_{2}} \psi_{c}^{2}
$$

and the barotropic zonal velocity by

$$
U_{b}=-\epsilon F_{D} / \beta_{0} \psi_{b y} .
$$

The term $w_{e}$, in (2.2a), is kept in view of deducing $U_{b}$ along $\Gamma$. The wind curl $w_{e}$ is taken to be a function of $y$ only so that $\Gamma$ is zonal and is the line where the net meridional Sverdrup transport vanishes. This assures the separation line between subpolar and subtropical gyre to be $\Gamma$.

In the following study, the Ekman pumping $w_{e}$ is chosen as a linear function of latitude:

$$
w_{e}=-w_{0}\left(1-y / y_{w}\right),
$$

where $y_{w}$ is the latitude where $w_{e}$ vanishes. This form is a crude approximation of the Ekman pumping of both the North Atlantic and North Pacific oceans near $\Gamma$.

The aim of this study focuses on solutions in which the mean zonal current opposes the Rossby wave phase propagation, that is, where $c_{R}=-U_{b}$ in Eq. (2.2b). We will therefore, before dealing with the full set of equations, investigate formally the dynamical behavior of these equations around this arrested wave condition.

\section{Midocean baroclinic boundary layers}

a. Dispersion relation near points where $c_{R}=-U_{c}$

With $r_{s}=0$ the behavior of the baroclinic flow satisfies the equation

$\psi_{c t}-\left[1+\epsilon_{1} \psi_{c}-\epsilon_{3} \psi_{c}^{2}-U_{b}(x)\right] \psi_{c x}-\epsilon_{2} \psi_{c x x t}=0$.

Here $\epsilon_{1}=\epsilon F_{D}\left(H_{1}-H_{2}\right) / H ; \epsilon_{2}=F_{D}^{-1} ; \epsilon_{3}=\left(\epsilon F_{D}\right)^{2}$ $\times H_{1} H_{2} /\left(H^{2}\right)$; and $U_{b}(x)$ is the zonal barotropic mean flow that includes the effect of a frictional western boundary layer, which allows the zonal-mean flow to vanish at the western wall. This layer will be dealt with in subsequent sections.

Suppose $\psi_{c}=\psi_{c 0}$ (local quasigeostrophic approximation) and $U_{b}$ to be constant in the wave speed. Substituting a wave-type solution $\psi_{c}=A \exp [i(k x-\omega t)]$ leads to the dispersion relation or phase speed:

$$
\frac{\omega}{k}=\frac{-\left[1+\epsilon_{1} \psi_{c 0}-\epsilon_{3} \psi_{c 0}^{2}-U_{b}\right]}{1+\epsilon_{2} k^{2}} .
$$

Since the term in brackets can change sign at some longitude; that is, Rossby wave phase speed can change sign due to the zonal-mean current. The phase can propagate in both directions, east or west, depending on whether we are in a region where $U_{b}>-c_{R}$ or in a region where $U_{b}<-c_{R}$ (Luyten and Stommel 1986). 
Energy too presents the same feature, as it can be seen in the group velocity:

$$
\frac{d \omega}{d k}=\frac{-\left[1+\epsilon_{1} \psi_{c 0}-\epsilon_{3} \psi_{c 0}^{2}-U_{b}\right]\left[1-\epsilon_{2} k^{2}\right]}{\left[1+\epsilon_{2} k^{2}\right]^{2}} .
$$

As shown in Fig. 2, in the region where $U_{b}(x)$ is small, long Rossby waves carry energy to the west, while short ones carry energy to the east. On the other side, in regions where $U_{b}(x)$ dominates the Rossby wave speed, long waves propagate energy to the east and short ones to the west. Energy can therefore be trapped at points where $U_{b}=-c_{R}$ allowing baroclinic interior layers to develop around such points, the short waves propagating away from these points being eroded by friction. It follows that barotropic and baroclinic boundary layers can be located at different places in the ocean, the barotropic one on the western coast and the baroclinic one shifted to the east.

In the following section, friction will be added into the baroclinic field to dissipate the short waves and to build up baroclinic sharp layers located in the interior of the ocean centered around points where the effective Rossby wave speed $c_{R}+U_{b}$ changes sign.

\section{b. Interior baroclinic layer} is

The equation for the steady baroclinic interior layer

$$
\left(1+\epsilon_{1} \psi_{c}-U_{b}(x)\right) \psi_{c x}+r_{s} \psi_{c x x}=0 \quad \epsilon_{3} \ll \epsilon_{1}
$$

if the quadratic term in the Rossby wave speed is neglected.

The barotropic mean current in the interior far away from the western coast is given by the Sverdrup constraint and is linear in $x$ with the prescribed wind (2.5) $\left(w_{e}=0\right.$ but $\left.U_{b} \sim \partial w_{e} / \partial y \neq 0\right)$.

Since we are looking for baroclinic interior layers, and since we are dealing with a large-scale wind field ( $U_{b}$ varies on the basin width scale), to solve this equa-
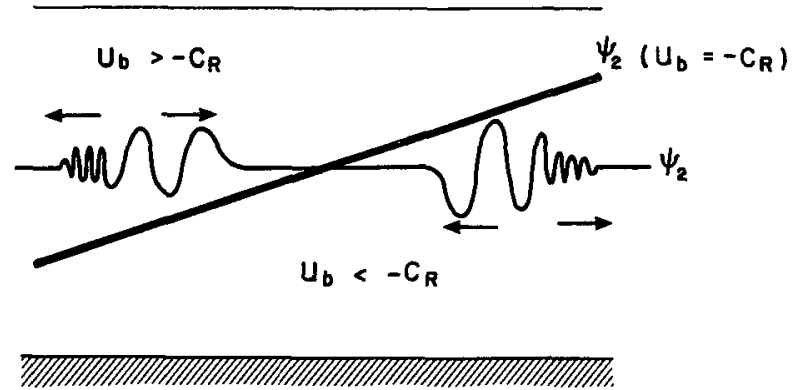

FIG. 2. A schematic east-west cross section displaying both the eastern regime where long Rossby waves propagate westward while short ones carry energy to the east and the western regime where propagations are reversed. The bold line represents the values of the interface displacement where the long baroclinic Rossby wave is arrested by the mean zonal current, line separating the eastern and western regime. The arrows give the directions of energy propagation. tion we assume that $U_{b}$ is constant in the vicinity of the interior layer. The interior layer has to be located around points where $U_{b}+c_{R}$ cancels. If we take, for example, a point where $h_{1}$ has the value $H_{1}$, then $U_{b}$ $=1$ since $c_{R}=-1$ in its nondimensionalized form for $h_{1}=H_{1}$. In the interior layer (3.4) then reduces to

$$
\epsilon_{1} \psi_{c} \psi_{c x}+r_{s} \psi_{c x x}=0 \text {. }
$$

Rescaling the length scale by $\delta=(x / l)$ leads to the equation

$$
\epsilon_{1} \psi_{c} \psi_{c \delta}+\frac{r_{s}}{1} \psi_{c \delta \delta}=0
$$

This balance is possible only if the two terms in Eq. (3.6) are of the same order, that is, if the length scale is of order

$$
1=\frac{-r_{s}}{\epsilon_{1}}
$$

remember that $\epsilon_{1}$ is negative for $H_{1}<H_{2}$. Formally, $l$ should be scaled as $-r_{s} /\left(\epsilon_{1} \psi_{c}\right)$ since the boundary-layer width depends on the amplitude of the interface displacement due to the nonlinear character of this interior layer. The width of this layer is inversely proportional to the amplitude, so we can expect, for a large interface displacement between east and west, to have a very sharp layer.

The equation to be solved with the aforementioned scaling is

$$
\psi_{c} \psi_{c \delta}-\psi_{c \delta \delta}=0
$$

which can be integrated to

$$
\frac{1}{2}\left(\psi_{c}^{2}\right)-\psi_{c \delta}=K
$$

With $K$ negative, the solution is given by

$$
\psi_{c}=-(-2 K)^{1 / 2} \operatorname{tg}\left[(-K / 2)^{1 / 2} \delta+K_{2}\right] ;
$$

$K_{2}$ is a constant taken to be zero to satisfy the condition $U_{b}=1$. This solution tends to infinity outside the boundary layer and therefore does not present a physically meaningful flow pattern. With $K$ positive, (3.8) can be integrated leading to the solution

$$
\psi_{c}=\frac{2 \psi_{c 1}}{K_{1} \exp \left(-\psi_{c 1} \delta\right)-1}+\psi_{c 1}, \quad \psi_{c 1}= \pm(2 K)^{1 / 2}
$$

with $\delta=\epsilon_{1} x /\left(-r_{s}\right)$.

The solutions (3.9) are shown in Fig. 3. The finite physical solution is the one with $K$ positive and $K_{1}$ negative ( $K_{1}=-1$ to satisfy the condition $\left.U_{b}=1\right)$. The nonlinear character of this boundary layer outcomes clearly in (3.9) and the width is given by $-r_{s} /$ $\left(\epsilon_{1} \psi_{c}\right)$. The solution is centered around the point where $U_{b}=-c_{R}$ and tends to constant values outside the boundary layer. Should the solution not be centered, the front would move, in the time-dependent problem, 


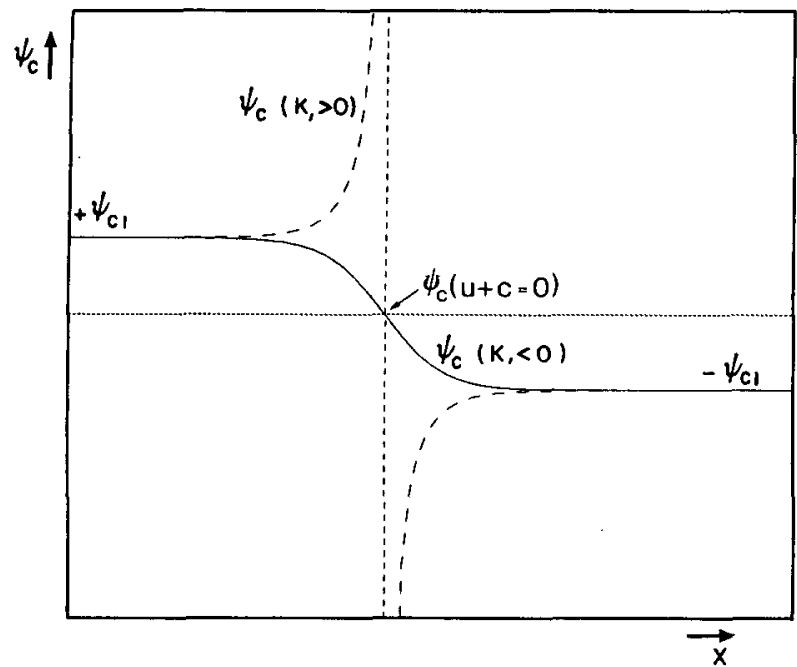

FIG. 3. Interface displacement for an interior frictional layer around a Rossby repellor. The physical finite solution retained is the continuous line centered around the Rossby repellor.

to a new Rossby repellor [Luyten and Stommel (1986) call the location where $U_{b}+c_{R}$ changes sign at $\Gamma$, the Rossby Repellor] where the solution presents a symmetric feature around this point. Figure 4 shows a spinup simulation, described later, building up such a boundary layer by imposing different boundary conditions on the eastern and western basin walls. The time-dependent dynamics is of the Burger equation- type, and the steady solution tends effectively to an arrested Taylor shock profile (Dodd et al. 1982) given by (3.9) centered around a Rossby repellor.

The principal goal of this paper is to match a western boundary layer to the northward communicating solution found in Schopp (1988) by imposing the same boundary value $\psi_{c}$ on east and west coasts so as to conserve heat content in each layer. Therefore, in the following section, we will add friction into the barotropic field to close the circulation on the western wall.

\section{Wind-driven deep western boundary layer}

\section{a. Barotropic boundary layer}

The barotropic steady dynamics is given by $(2.2 \mathrm{a})$ and the baroclinic inviscid equation by (3.1), which has steady solutions given by

$$
\psi_{c}=\text { const }
$$

and the arrested Rossby wave $\psi_{c}^{+}$and $\psi_{c}^{-}$of the following quadratic equation:

$$
1+\epsilon_{1} \psi_{c}-\epsilon_{3} \psi_{c}^{2}-U_{b}(x)=0
$$

If we take a Rayleigh frictional term $(R=0)$, an approximated barotropic boundary-layer solution of (2.2a) will be

$$
\psi_{b}=\psi_{b i}\left[1-\exp \left(-\frac{\left(x-x_{w}\right) L}{l}\right)\right],
$$

where $R_{s}=l / L$ is the ratio of the boundary-layer length

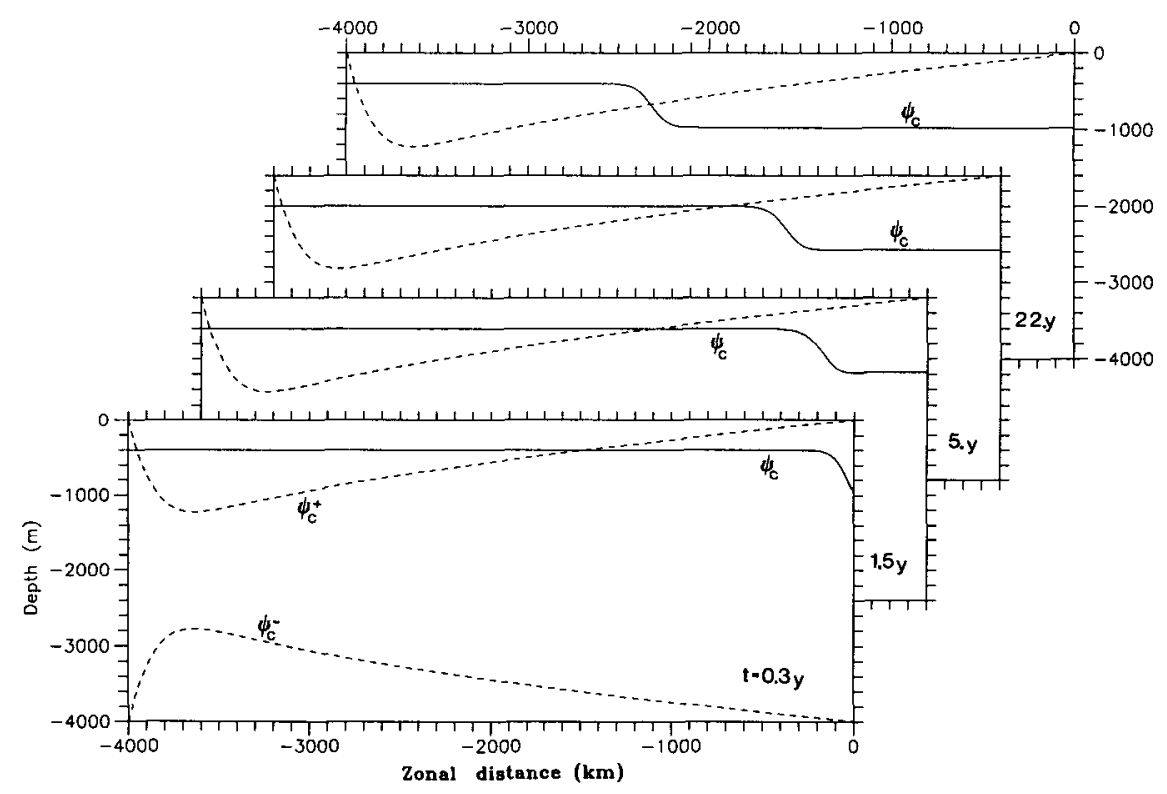

Fig. 4. East-west cross section showing a spinup process of an interior frictional boundary layer. The stippled line represents the depth of the interface where the mean zonal current cancels out the Rossby wave speed. The final steady solution is located where the interface is symmetrically centered around the arrested solution $\left(L=200 \mathrm{~km}, U=2 \mathrm{~cm} \mathrm{~s}^{-1}, H_{1}=400 \mathrm{~m}, H_{2}=3600 \mathrm{~m}\right.$, latitude $\left.=48^{\circ} \mathrm{N}, g^{\prime}=0.015, L_{D}=21 \mathrm{~km}, w_{0}=0.0001 \mathrm{~cm} \mathrm{~s}^{-1}\right)$. 
scale $l$ to the interior length scale $L$, and where $x_{w}$ represents the location of the western wall. In (4.2), the interior Sverdrup solution $\psi_{b i}$ is given by

$$
\psi_{b i}=\frac{w_{e}(y)}{\epsilon \beta_{0} f H}\left(x-x_{e}\right),
$$

where $x_{e}$ is the longitude of the eastern wall. Equation (4.2) leads then to the zonal barotropic velocity along $\Gamma:$

$$
\begin{aligned}
U_{b}(x)=-\frac{\epsilon F_{D}}{\beta_{0}} \psi_{b y} & =-\frac{\epsilon F_{D}}{\beta_{0}} \psi_{b i y} \\
\times & {\left[1-\exp \left(-\frac{\left(x-x_{w}\right) L}{l}\right)\right] . }
\end{aligned}
$$

Substituted into (4.1b), one obtains the two steady solutions for the baroclinic mode $\psi_{c}$, where $c_{R}$ $=-U_{b}(x)$. Figure 5 shows such solutions for different parameters. An east-west cross section along $\Gamma$ is represented with the interface displacement for the two solutions $\psi_{c}^{+}$and $\psi_{c}^{-}$.
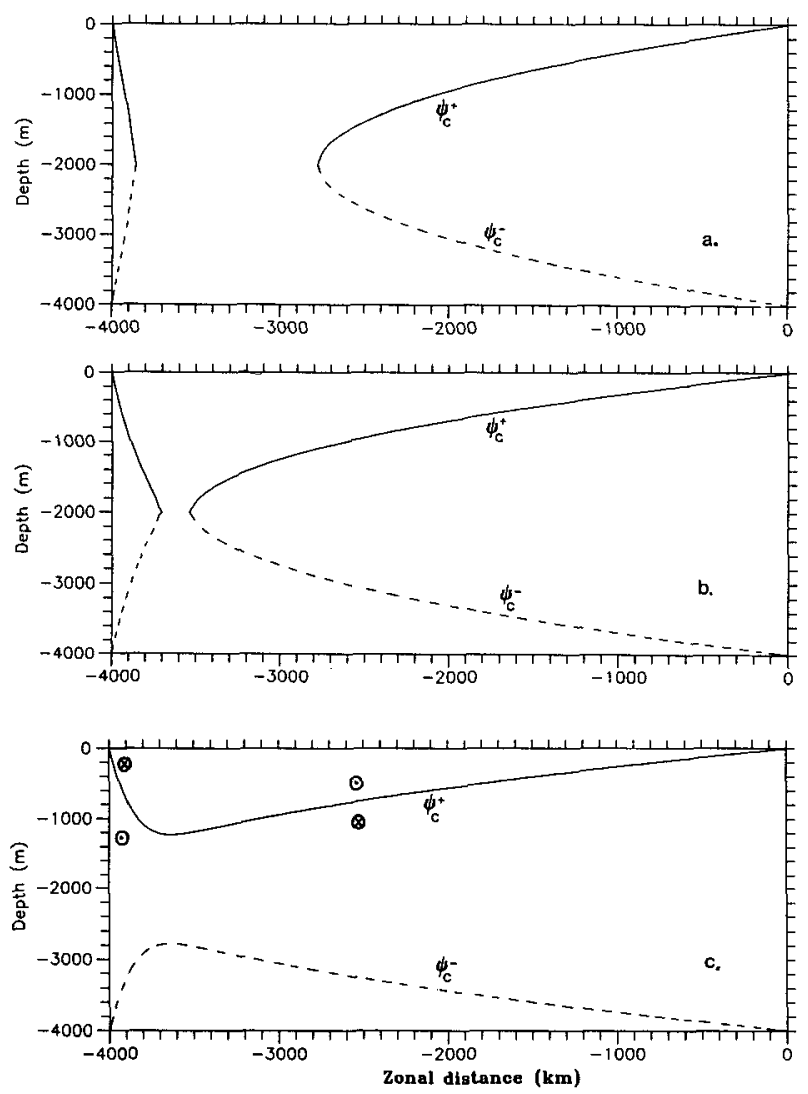

Fig. 5. East-west cross section representing the interface location in the case $u=-c_{R}$. The stippled line represents the second solution of the quadratic equation $\left(h_{1}>h_{2}\right)$. (a) $L=200 \mathrm{~km}, U=2 \mathrm{~cm} \mathrm{~s}^{-1}$, $H_{1}=400 \mathrm{~m}, H_{2}=3600 \mathrm{~m}$, latitude $=48^{\circ} \mathrm{N}, g^{\prime}=0.01, L_{D}=21$ $\mathrm{km}, w_{0}=0.0001 \mathrm{~cm} \mathrm{~s}^{-1}$. (b) Same as in (a) but with latitude $=51.5^{\circ} \mathrm{N}$ and $g^{\prime}=0.015$. (c) Same as in (a) but with $g^{\prime}=0.015$.
In Fig. 5a and in the eastern part of the basin one notices a parabola representing the two solutions satisfying the arrested wave condition ( $4.1 \mathrm{~b}$ ); the Rossby wave speed $c_{R}$, via the interface height $\psi_{c}$ adjusts itself so as to cancel exactly the eastward-increasing mean zonal current $U_{b}(x)$. They are both solutions found by Pedlosky (1984), Schopp and Arhan (1986), and Schopp (1988). As noted in these papers, the western limit of the parabola is the limit where the Rossby wave speed reaches its maximum $\left(h_{1}=h_{2}\right)$. Farther west, the zonal-mean current is still increasing westward and can no longer be in balance with the wave speed. On the western side of the basin and in the boundary layer, the barotropic zonal current decreases and is able again to find values of $c_{R}$ to cancel out until it reaches the no-normal flux along the wall. Therefore, as observed in this figure, two solutions of arrested wave type are again possible on the western side.

The zonal widths of these regions of existing arrested waves are a function of different parameters affecting both the Rossby wave speed and the mean current. Increasing the wind-stress curl would shrink the eastern portion, $U_{b}$ being stronger; the maximum Rossby wave speed would be reached closer to the eastern boundary. Also varying the depth of the ocean, the latitude of $\Gamma$ or $\Delta \rho / \rho$ would change the Rossby wave speed and therefore change the extension of these regions.

Some examples are given in Fig. 5 with a Rayleigh frictional boundary layer. One can observe, by affecting the Rossby wave speed, that the western and eastern arrested wave regions slowly match together by increasing $f$ or decreasing $\Delta \rho / \rho$ (Figs. $5 b, c$ ), the matching condition of both regions being given by the following condition: $U_{b}$ (maximum in the basin) $\leqslant-c_{R}$ (maximum $h_{1}=h_{2}$ ).

The resulting upper solution $\psi_{c}^{+}$in Fig. $5 \mathrm{c}$ is appealing since it can be compared with the zonal structure of the actual thermocline in the real ocean: a slowly westward deepening across the basin followed by an abrupt outcropping near the western boundary. A deep western southward current is observed in this solution with deep northward flow in the interior. This baroclinic boundary layer has the same extension as the barotropic one. One can notice here that these solutions do not depend on the choice of $H_{1}$ and $H_{2}$ (but depend on $H$ ), a result issued from the presence of the nonlinear terms in the wave speed. Thus, if this solution is stable, it would be able to give another physical process for the observed deep current in the western boundary layer. Remember that this current is only forced by wind and not by interfacial cross-isopycnal flux of water as it is in the Stommel and Arons model.

It is difficult to justify the friction type used since we are in a stratified ocean. The choice of such a frictional boundary layer has only been taken for process studies. A more realistic friction type would require use a Munk-type boundary layer. To show that the use of a Munk layer gives the same type of behavior, we 
have computed a solution with a Munk boundary layer. The barotropic zonal current along $\Gamma$ has been obtained by the boundary-layer approach from (4.1) with $R_{s}$ $=0$ and is given by

$$
\begin{aligned}
-\psi_{b y}= & -\psi_{b i y}\left(1-\exp \left(-\frac{\left(x-x_{w}\right) L}{2 l}\right)\right. \\
\times & {\left[\cos \left(\frac{\sqrt{3}\left(x-x_{w}\right) L}{2 l}\right)\right.} \\
& \left.\left.+\frac{1}{\sqrt{3}} \sin \left(\frac{\sqrt{3}\left(x-x_{b}\right) L}{2 l}\right)\right]\right\} .
\end{aligned}
$$

Figure 6 shows the same matching of eastern and western arrested wave solutions.

The next section deals with the stability of such solutions and shows that these solutions are unstable in the western boundary layer where the barotropic mean current increases toward the east. Furthermore, time dependency is added and transients are computed principally to know the states toward which these solutions evolve.

\section{b. Unstable solution}

Neglecting the quadratic term in the Rossby wave speed, the baroclinic equation can be written as

$$
\psi_{c t}-\left[1+\epsilon_{1} \psi_{c}-U_{b}(x)\right] \psi_{c x}-\epsilon_{2} \psi_{c x x t}=r_{s} \psi_{c x x} \text {. }
$$

With no friction, $r_{s}=0$, the interface displacement $\psi_{c s}=\left(U_{b}-1\right) / \epsilon_{1}$ where $c_{R}=-U_{b}$ satisfies this equation and is represented in Fig. 7. The initial conditions have been taken so as to have finite depth at the eastern and western walls. We define then a finite amplitude perturbation from the state $\psi_{c s}$ :

$$
\psi_{c}=\psi_{c s}+\psi
$$

Substituting into Eq. (4.6) with $r_{s}=0$, one obtains

$$
\psi_{t}-\epsilon_{1} \psi \psi_{x}-\frac{\partial U_{b}}{\partial x} \psi-\epsilon_{2} \psi_{x x t}=0
$$

(I)

(II)

The presence of an exponential growing term II in

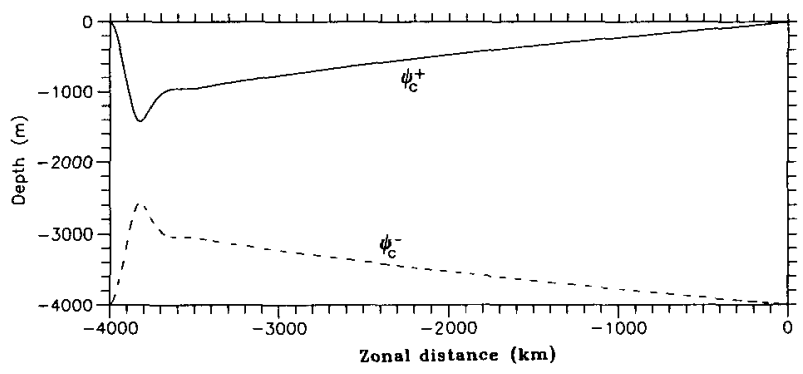

FIG. 6. Same as in Fig. 5 but with a Munk frictional boundary layer.

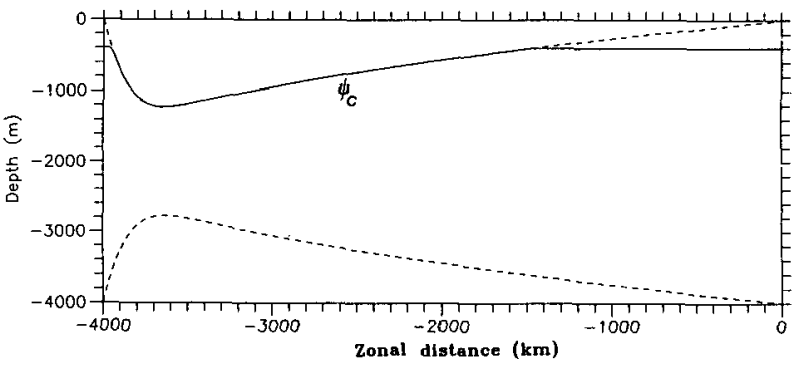

FIG. 7. Initial steady condition. No friction is present in the baroclinic field.

this equation shows that solution $\psi_{c s}$ is stable if $\partial U_{b} /$ $\partial x$ is negative, that is, outside the western boundary layer. This stability problem can be interpreted in terms of Rossby wave propagation. In the stable region long Rossby waves propagate energy toward the arrested solution, whereas in the unstable region they propagate energy quickly away.

If friction is neglected and initially the ocean is in the state where $c_{R}=-U_{b}$ (Fig. 7), a slight perturbation in the western boundary would lead to a transient regime evolving from this state toward a more stable one. Furthermore, the nonlinear propagating term I in (4.7) shows that, if positive, the perturbation would evolve toward the east of the basin and, if negative, toward the west. Therefore one can expect that, depending on the sign of the perturbation, the solution $c_{R}=-U_{b}$ will evolve toward different states, the solution $\psi_{c s}$ (Fig. 7) being at the boundary between two more stable ones. Figure 8 shows the evolution of the interface from the solution $\psi_{c s}$. With a small negative perturbation, the interface propagates toward the west under the effect of long waves. When this front reaches the western boundary, short waves are reflected while propagating energy to the east. One might expect that in adding friction into the baroclinic field, these short waves, generated at the western wall, will be eroded to form a baroclinic boundary layer.

\section{c. Evolution toward communication with a deep western boundary current}

Including friction in the baroclinic mode no longer allows the interface $\psi_{c s}\left(U_{b}=-c_{R}\right)$ to be a solution of equation:

$$
\begin{aligned}
\psi_{c t}-\left[1+\epsilon_{1} \psi_{c}-\epsilon_{3} \psi_{c}^{2}-U_{b}(x)\right] \psi_{c x} & \\
& -\epsilon_{2} \psi_{c x x t}=r_{s} \psi_{c x x} .
\end{aligned}
$$

In the interior, where friction has only a small effect, this added term does not affect this particular solution, but in the western boundary layer where sharp fronts are present, this term displaces the unstable solution to the west, and one expects then that the perturbation from the arrested wave solution has to be larger to pro- 


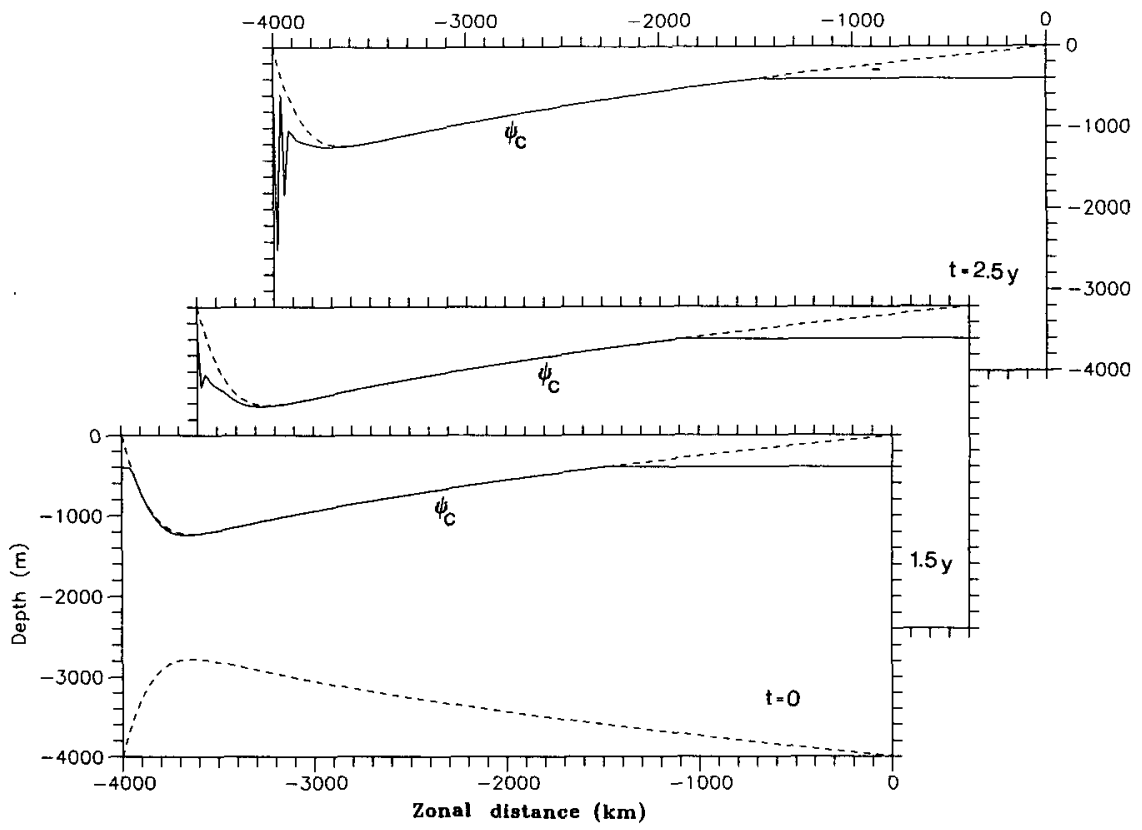

FIG. 8. Case with a slight negative perturbation showing the unstable character of this particular steady solution. As the front propagates to the western coast short waves are reflected. The parameters are the same as in Fig. 5c with $h_{1}\left(x_{e}\right)=h_{1}\left(x_{w}\right)=400 \mathrm{~m}$.

duce a transient regime traveling to the west, but overall, this westward shifting does not affect qualitatively the physics underlying the solution presented.

Figure 9 shows the evolution of the interface, initially in the state of communication with a slight positive perturbation, the western front propagates to the east wanting to produce solitary waves, but friction, present in this run, erodes them, leaving only a flat interface behind. The front propagating to the east is of a Taylor shock-type front resulting from the balance due to

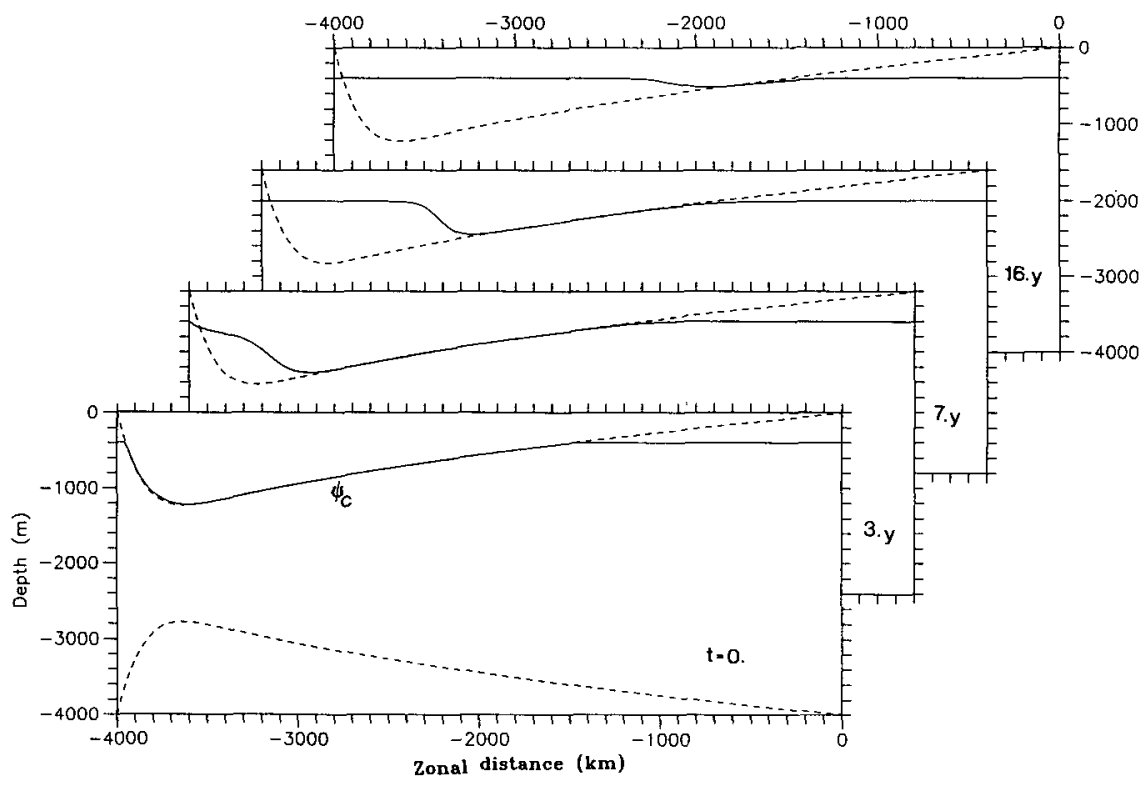

FIG. 9. The case with friction in the baroclinic field and a positive perturbation. Evolution toward the noncommunicating solution where the interface is zonally flat. Friction here erodes smaller-scale features like solitary waves. Same parameters as in Fig. 8. 
steepening (nonlinearity) and diffusion. In a few years, the front has traveled across the basin leaving behind an ocean without transfer of water between the gyres.

A small negative perturbation would produce the same behavior since we are still in the attraction of the no cross-gyre flow stable state, diffusion having shifted the unstable steady solution to the west. A greater negative perturbation, however, shows that there is a second possible solution situated in the west. The front as seen in Fig. 10, travels to the west, and now instead of reflecting short waves while reaching the coast, builds up a narrow baroclinic boundary layer near the coast diffusing the short waves by friction. One can notice that the baroclinic boundary layer is included in the barotropic one. Would this not be the case, the second stable solution would not be possible since whatever the magnitude of the initial condition would be, the interface would still be in the region where $U_{b}+c_{R}$ $>0$ and evolve toward the noncommunicating solution.

One can notice that the steady crossing solution (Fig. 10 ) can be easily destroyed in perturbing the westernmost part of the interface. For example, injection of cold water on the western coast would bring the interface into the noncommunicating solution's region, where $U_{b}+c_{R}>0$, forcing the interface to evolve like the solution in Fig. 9. How is it then possible to go back into the original flow pattern? This can be achieved by including cross-isopycnal upwelling (equivalent to a heating function) into the dynamics over a small region in the western boundary layer overlapping the arrested solution. The resulting deepening of the interface produced by upwelling spreads east and west into nonforced regions, being carried by the long Rossby waves. Figure 11 shows such a simulation in which the noncommunicating solution evolves toward the communicating solution.

We will evaluate the magnitude of the undercurrent found in the communicating solution. Applying the geostrophic relation $\rho_{0} f v_{1}=\rho_{1} g h_{x}$ and $\rho_{0} f v_{2}=\rho_{1} g h_{x}$ $+\left(\rho_{2}-\rho_{1}\right) g h_{2 x}$ and the Sverdrup constraint along $\Gamma$, $h_{1} v_{1}+h_{2} v_{2}=0$, for this two layer model, the meridional deep layer transport is obtained by

$$
h_{2} v_{2}=\frac{g^{\prime}}{f}\left(h_{2}-\frac{h_{2}^{2}}{H}\right) \frac{\partial h_{2}}{\partial x},
$$

where $h$ has been replaced by the mean depth of the ocean $H$.

Equation (4.9), integrated over the western boundary layer, gives the magnitude of the undercurrent, which is

$$
\int_{x_{w}}^{x_{b}} h_{2} v_{2} d x=\frac{g^{\prime}}{f}\left(\frac{h_{2}^{2}}{2}-\frac{h_{2}^{3}}{3 H}\right)_{h_{2}\left(x_{w}\right)}^{h_{2}\left(x_{b}\right)},
$$

where $h_{2}\left(x_{w}\right)$ is the thickness of the deep layer at the western wall and $h_{2}\left(x_{b}\right)$ is its thickness on the eastern limit of the boundary layer.

The total transport depends only on the difference of $h_{2}$ on both sides of the undercurrent. The smaller $h_{2}\left(x_{b}\right)$ is compared to $h_{2}\left(x_{w}\right)$ and the stronger is the magnitude of the undercurrent. To relate this transport to the forcing and model parameters, one needs to es-

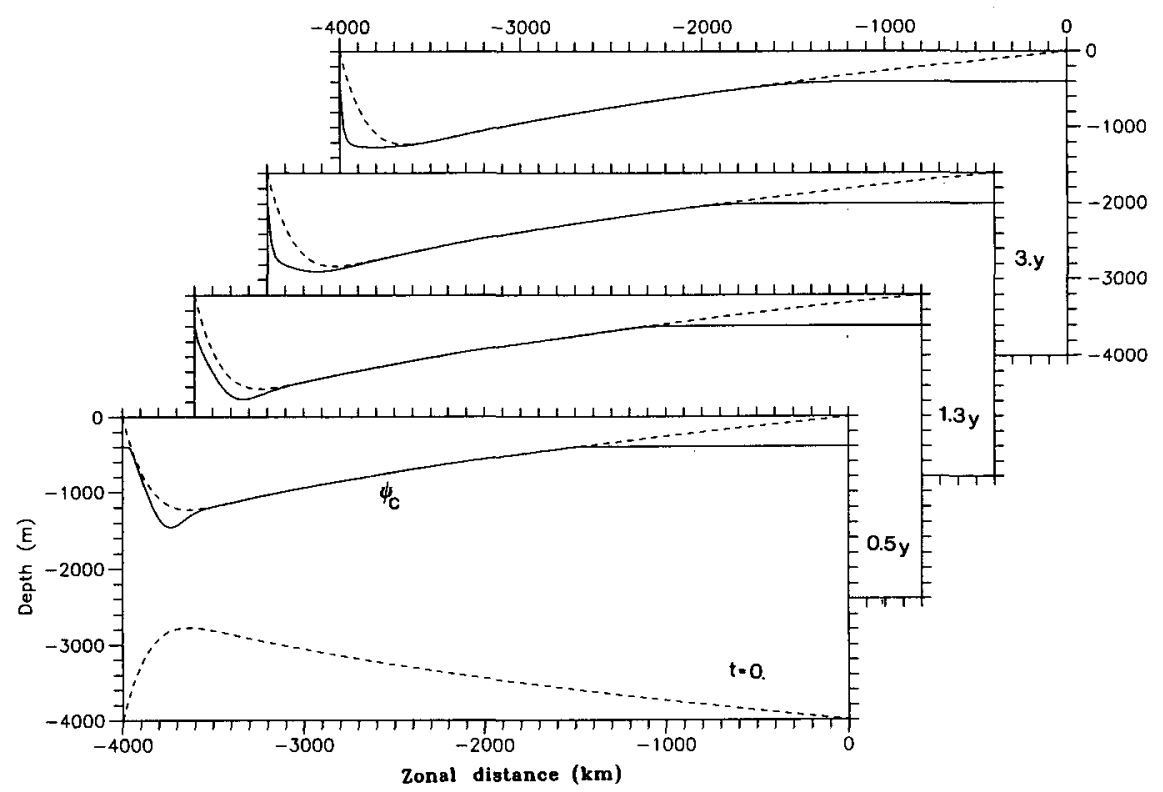

Fig. 10. Case with a negative perturbation. Evolution toward a stable cross-gyre flow solution. Northward flow (southward flow) in the interior deep layer (upper layer) with a strong southward (northward) jet in the western boundary layer. Same parameters as in Fig. 8. 


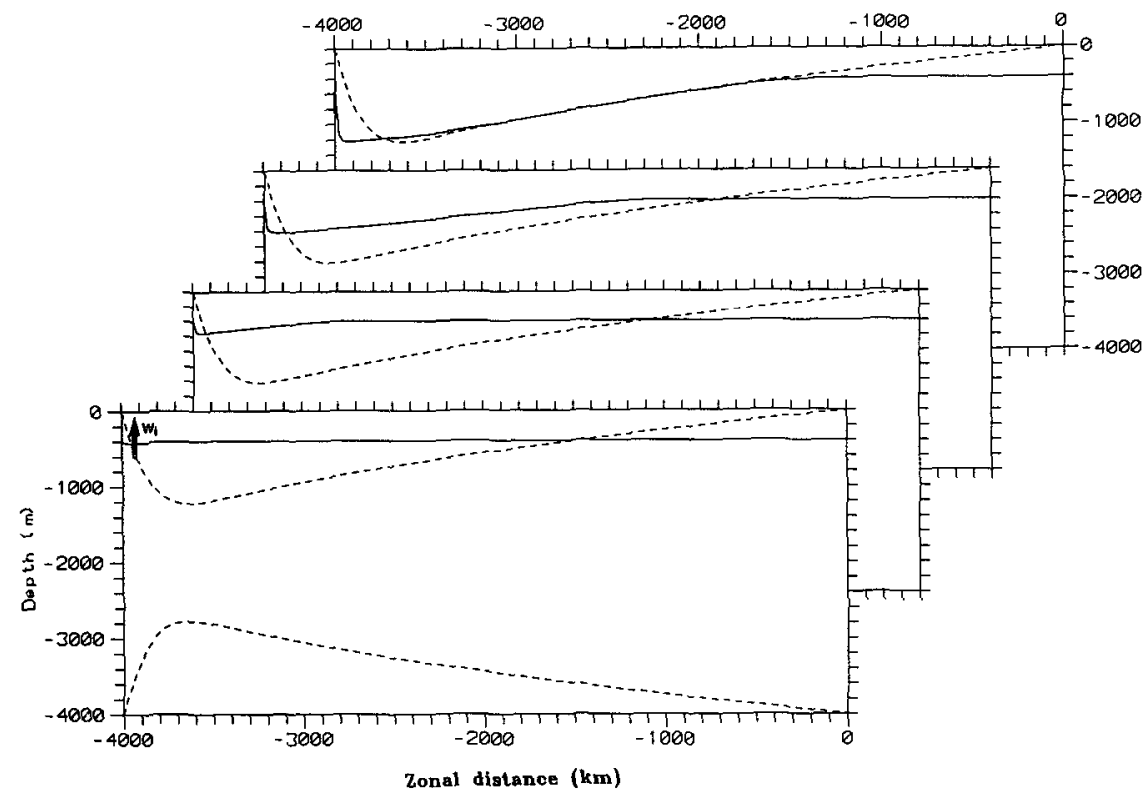

FIG. 11. Case including positive cross-isopycnal upwelling over a small region in the western part of the basin allowing the noncommunicating solution to evolve toward the communicating solution.

timate $h_{2}\left(x_{b}\right)$. This can be easily done by using the arrested wave condition $U_{b}=-c_{R}$. In dimensional form, $c_{R}=-\left(\beta g^{\prime} / f^{2}\right)\left[\left(H-h_{2}\right) h_{2} / h\right]$ and $U_{b}(x)$ $=-[f /(\beta h)]\left(x-x_{e}\right) w_{e y}$ for the interior solution. The depth of the thermocline will be deepest where $c_{R}$ is the greatest; that is, $h_{2}\left(x_{b}\right)$ is obtained where $U_{b}$ reaches its maximum, that is, near the western boundary. An estimation of $h_{2}\left(x_{b}\right)$ is given by solving the arrested condition for $h_{2}$, which gives

$$
h_{2}\left(x_{b}\right)=\frac{H}{2}+\frac{H}{2}\left(1-\frac{4 f^{3} L_{1} w_{0}}{H^{2} \beta^{2} g^{\prime} L_{2}}\right)^{1 / 2},
$$

where $L_{1}$ is the zonal width of the interior solution and $L_{2}$ the meridional scale of the wind. With the parameters used in Fig. $5 \mathrm{c}$, with $L_{1}=3500 \mathrm{~km}$ and $L_{2}=200$ $\mathrm{km}$, the value of $h_{2}\left(x_{b}\right)$ is $2800 \mathrm{~m}$, consistent with the value in Fig. 5c. With $h_{2}\left(x_{w}\right)=3600 \mathrm{~m}$ and $h_{2}\left(x_{b}\right)$ $=2800 \mathrm{~m}$, one obtains a transport of around $-70.10^{6}$ $\mathrm{m}^{3} \mathrm{~s}^{-1}$. The observed value of the magnitude of this undercurrent in the Atlantic Ocean is of order -10 to $-20\left(\times 10^{6} \mathrm{~m}^{3} \mathrm{~s}^{-1}\right)$. Reducing the wind strength $w_{0}$, increasing the meridional scale $L_{2}$ of the wind, or reducing the zonal width $L_{1}$ of the basin would decrease $U_{b}$ and increase $h_{2}\left(x_{b}\right)$ in Eq. (4.11) and therefore would reduce the transport of the undercurrent. For example, if $L_{2}=300 \mathrm{~km}$, the transport would shrink to $-20 \times 10^{6} \mathrm{~m}^{3} \mathrm{~s}^{-1}$; a value more comparable to the observed in the Atlantic. The high value used in this work is only for the sake of process studies.

\section{Barotropic interior layer}

a. Formation of "cold" and "warm eddies" near the front, weak friction

Suppose now we shift one part of the barotropic boundary layer into the interior of the basin, forcing the existence of barotropic interior layer dynamics in the middle of the gyre. That such a layer exists is not obvious; nevertheless Parsons (1969) and Huang (1984) have presented solutions for large-scale flow including cross-gyre flow with a Gulf Stream crossing $\Gamma$ in the middle of the basin. To simulate such an interior layer, the preceding $U_{b}(x)$ has been modified. Figure 12 shows the initial solution $\psi_{c s}\left(U_{b}=-c_{R}\right)$ exhibiting an interior barotropic boundary layer (dashed line). At the front where instability is present, that is, where $U_{b}$ increases with $x$, the interface is forced to evolve in a manner that is dependent on the perturbation involved. A positive perturbation applied to this initial state will propagate to the east while a negative one will propagate to the west. With no friction in the baroclinic mode, $r_{s}=0$, the resulting flow pattern will favor solitary waves. Figure 12a shows this outbreak of solitary waves from the front; a positive perturbation will lead to "cyclonic" solitons in the upper layer able to represent cold "eddies" propagating eastward. On the contrary, if a negative perturbation is applied near the front (Fig. 12b), "anticyclonic" solitons emerge and propagate in the opposite direction, here to the west where the upper layer is shallower. They are able 

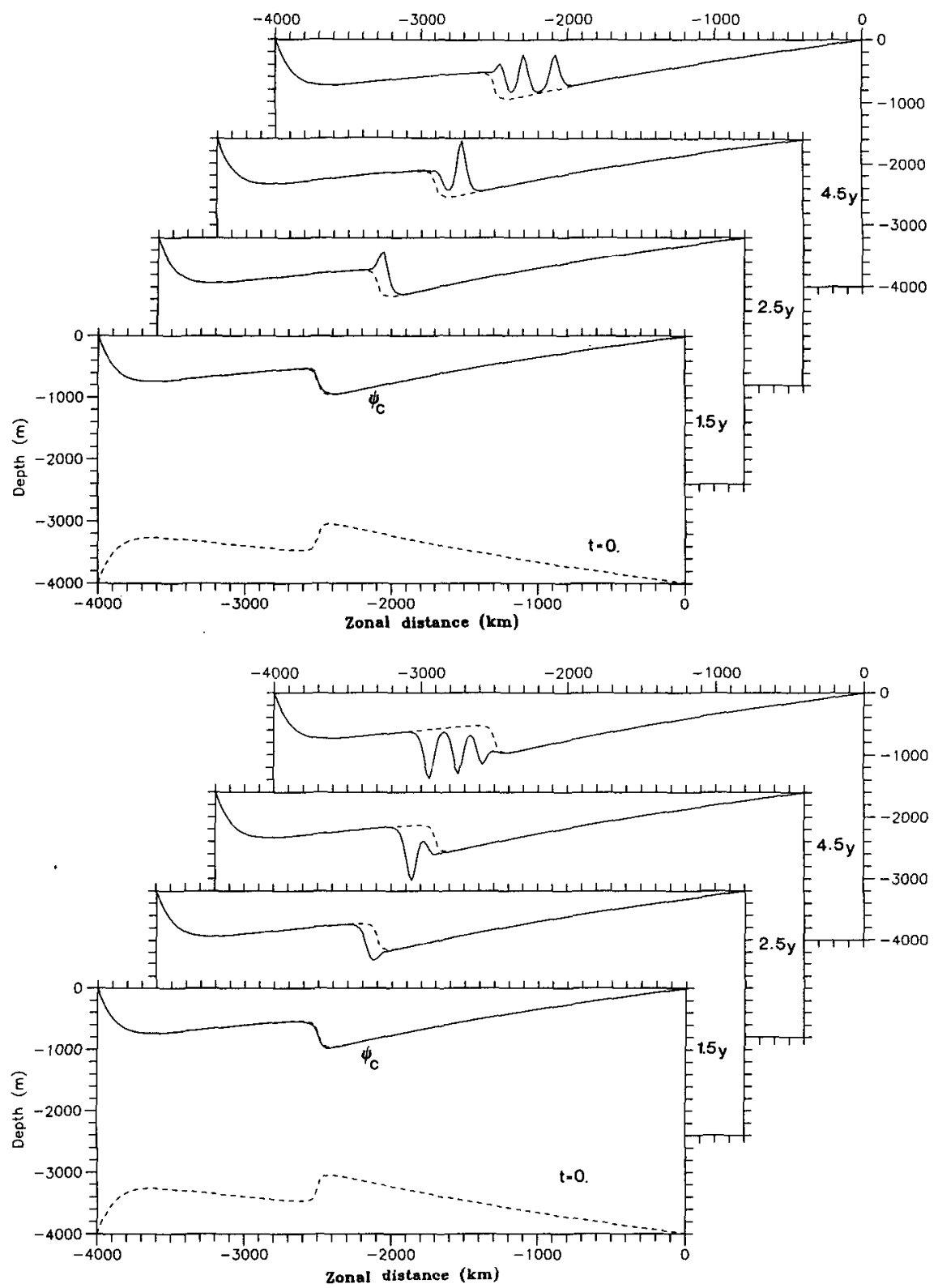

FIG. 12. Case with an interior barotropic layer and no friction in the baroclinic field. (a) A positive perturbation generates the eastward propagation of solitary waves (cold rings). (b) A negative perturbation generates the westward propagation of solitary waves (warm rings).

to represent warm eddies propagating into colder water. These waves could thus be one way in which cold rings and warm eddies are generated along the Gulf Stream.

\section{b. Formation of a baroclinic front, strong friction}

In adding friction to the baroclinic mode, instead of solitary waves appearing, we observe propagating fronts. Figure 13a illustrates such evolving interfaces. Initially a negative perturbation of the interface has been applied to the arrested Rossby wave solution at the interior boundary layer. One observes an eastward traveling front near the western coast. In the remaining part of the boundary layer, the interface propagates westward. When the two opposite-traveling fronts come together they combine nonlinearly and propagate in the direction where they are able to build up a symmetric front, of the type described in section $3 \mathrm{~b}$. The final steady state is stable and one observes a deepening of the thermocline in the eastern part followed by an abrupt outcropping. If the centered profile were not realizable, the interface would evolve toward a different state. Figure $13 \mathrm{~b}$ represents a case in which the barotropic field and the perturbations imposed are such 

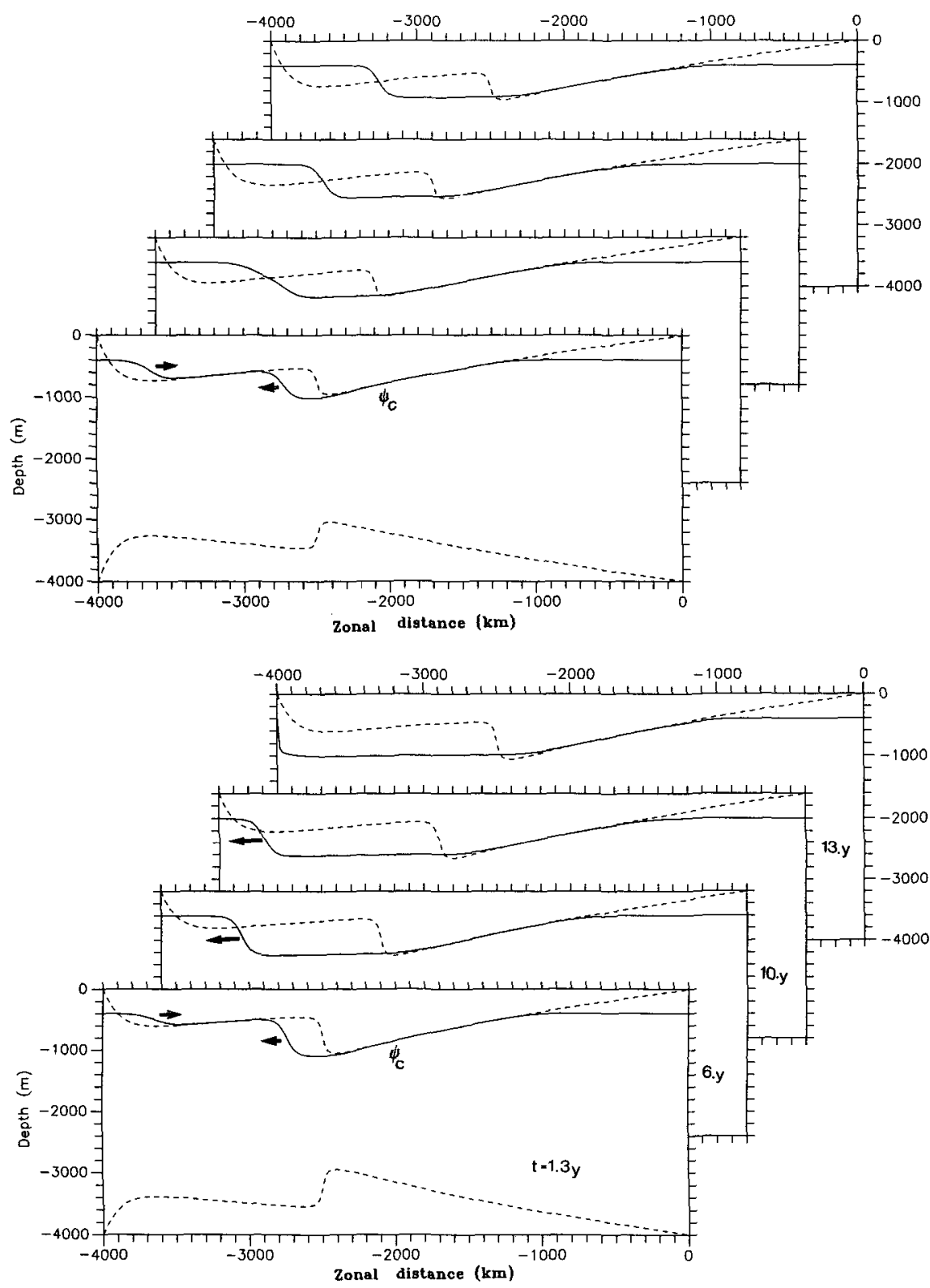

FiG. 13. Case with an interior barotropic layer and friction in the baroclinic field. (a) Case where the baroclinic layer is situated in the interior of the basin. The steady solution exhibits a symmetric feature around the Rossby repellor. (b) Case where the baroclinic boundary layer is localized at the coast. No Rossby repellor that exhibits an asymmetric solution exists.

that the solution cannot reach a final state with an interface symmetrically positioned around a Rossby repellor. Therefore, the solution propagates in the direction depending on the amplitude of the fronts, here to the west.

\section{Discussion}

A simple two-layer Sverdrup-type model completed with a frictional western boundary layer has been investigated along the zero wind-stress curl line $\Gamma$. The effect of large excursions of isopycnals has been taken into account to allow Rossby wave phase speed to vary with layer thicknesses. The resulting pressure drag on isopycnals, responsible for westward propagating Rossby waves, is therefore able to vary and to counteract the pressure drag term due to the varying zonal Sverdrup current acting on isopycnals. If both are exactly in balance all along $\Gamma$, an arrested Rossby wave solution results with zonally varying interface depth leading to a baroclinic cross-gyre flow pattern able to transfer water carrying heat and salt from one gyre to the other. In the western frictional boundary layer, where the interface outcrops, the solution exhibits an 
unstable character. Transients show that the arrested wave is located at the threshold of two more stable flow patterns. Depending on the sign of the perturbation, the arrested current system will evolve and be attracted to one or the other of the two adjacent stable steady solutions. One of these solutions displays a deep slow northward flow in the basin's interior extended by a deep strong southward current on the western wall. The upper-layer circulation displays a reversed current system. The other solution is the one where the interface is zonally flat all across the ocean and therefore does not allow water to cross the gyre separation line.

It is interesting to speculate on the possible relationship between the solutions obtained here with the circulation in the North Atlantic and North Pacific oceans. In the upper and intermediate layers of the North Pacific, subpolar and subtropical gyres exchange properties only by diffusive processes (Reid 1965), while in the North Atlantic warm salty waters flow into the subpolar gyre. This seems to have large consequences for convection processes and water-mass formation. The Atlantic, on the other hand, displays a singular flow field in that it exhibits deep water mass formation. A deep strong current carrying surface waters formed at a higher latitude is observed on the western wall crossing $\Gamma$. It is admitted that this water mass flows slowly back to its source in the basin's interior. It was Stommel and Arons (1960), in including crossisopycnal fluxes into Sverdrup-type models, who predicted such a circulation pattern. In the model studied here, the solution with cross-gyre flow could also contribute to such an observed deep flow pattern along $\Gamma$.

Schopp and Arhan (1986) showed that in the threedimensional gyre communicating problem, the warm water layer extends into the subpolar gyre with a deep layer outcropping farther north. Changing the wind strength or modifying the heat content would shrink or enlarge the extent of this warm layer in the subpolar gyre. Should by this process, the stable steady crossgyre flow brought into the region where Rossby waves propagate to the east, for example, in moving the interface on the western boundary across the unstable solution, the flow field would evolve toward the noncommunicating solution by recovering the high-latitude gyre with light waters. The window, where deep layer outcrops, maintained by the cross-gyre flow pattern, would therefore be shielded from the atmosphere and deep-water formation would be stopped. This would rather be like the case of the Pacific Ocean where light waters cover the subpolar gyre and inhibit deep convection and therefore deep-water formation. To give some weight to this scenario, Warren's (1983) paper titled "Why is there no water formation in the North Pacific" gives us some help. The concluding answer to the question that the author raised was: there is a flow of salty warm water from the subtropical gyre into the subpolar gyre in the Atlantic Ocean that allows the thermocline to be weak and eases convection pro- cesses, a mechanism not present in the Pacific where the presence of a strong halocline inhibits destruction of the density gradient. It seems to be necessary to have cross-gyre flow to carry warm, salty waters into higher latitudes to trigger convection processes. The model proposed here has most of these ingredients. Furthermore, it also has a deep layer surfacing at high latitudes where convection can be easily activated.

Nevertheless, what triggers the cross-gyre flow? Is it convection that builds such a cross-gyre flow system or is it cross-gyre flow that activates convective processes? One interpretation would be to give both as much importance: cross-gyre flow is locally mechanically forced but is maintained by thermodynamic processes farther north.

Acknowledgments. The author was supported in this investigation by CNRS. One part of this work has also been supported by the School of Oceanography, University of Washington, Seattle, during the author's stay with Peter Rhines. Useful discussions of the manuscript with Alain Colin de Verdiere are acknowledged. I wish to also acknowledge an anonymous reviewer whose careful reading helped correct numerous small errors in the text.

\section{REFERENCES}

Charney, J. G., and G. R. Flierl, 1981: Oceanic analogues of large scale atmospheric motions. Evolution in Physical Oceanography, B. A. Warren and C. Wunsch, Eds. The MIT Press, 504-548.

Dewar, W. D., 1987: Planetary shock waves. J. Phys. Oceanogr., 13, $470-482$.

Dodd, R. K., J. C. Eilbeck, J. D. Gibbon, and H. C. Morris, 1982 Solitons and Nonlinear Wave Equation. Academic Press, 630 pp.

Huang, R. X., 1984: The thermocline and current structure in subtropical/subpolar basins. Doctoral dissertation, Massachusetts Institute of Technology, WHOI-84-42, $218 \mathrm{pp}$.

Luyten, J., and H. Stommel, 1986: Gyres driven by combined wind and buoyancy flux. J. Phys. Oceanogr., 16, 1551-1560.

Luyten, J., J. Pedlosky, and H. Stommel, 1983: The ventilated thermocline. J. Phys. Oceanogr., 13, 292-309.

Parsons, A. T., 1969: A two-layer model of Gulf Stream separation. J. Fluid Mech., 39(Part 3), 511-528.

Pedlosky, J., 1984: Cross-gyre ventilation of the subtropical gyre: An internal mode in the ventilated thermocline. J. Phys. Oceanogr., 14, 1172-1178.

Reid, J. L., 1965: Intermediate waters of the Pacific Ocean. The Johns Hopkins Oceanographic Studies, Vol. 2, 85 pp.

Rhines, P. B., and W. Young, 1982: A theory of the wind driven circulation, I: Mid-ocean gyres. J. Mar. Res., 40(Suppl.), 559596.

Schopp, R., 1988: Spinup toward communication between large oceanic subpolar and subtropical gyres. J. Phys. Oceanogr., 18, 1241-1259.

Schopp, R., and M. Arhan, 1986: A ventilated middepth circulation model for the eastern North Atlantic. J. Phys. Oceanogr., 16, 344-357.

Stommel, H., and A. Arons, 1960: On the abyssal circulation of the World Ocean-I. Stationary planetary flow patterns on a sphere. Deep-Sea Res., 6, 140-154.

Warren, B. A., 1983: Why is there no water formation in the North Pacific? J. Mar. Res., 41(2), 327-347. 